MINI-SYMPOSIUM

\title{
Management of microvascular dysfunction and reperfusion injury
}

\section{A Prasad, B J Gersh}

$\mathrm{T}$ he aim of reperfusion therapy for acute myocardial infarction (AMI) is to rapidly restore coronary blood flow and myocardial perfusion with the objective of salvaging myocardium. The treatment of AMI for many years has focused on achieving patency of the conduit epicardial artery at the site of plaque rupture and occlusive thrombus. Major advances in interventional techniques and adjunctive pharmacological treatment have made it possible to achieve normal (TIMI grade 3 ) epicardial flow in approximately $95 \%$ of patients. ${ }^{1}$ This success has also highlighted the limitations of current treatment with regards to the goal of restoring myocardial perfusion. A significant proportion of patients, perhaps as many as $40 \%$, do not regain microvascular and myocardial perfusion despite the restoration of TIMI grade 3 flow. ${ }^{12}$ The major source of these data has been acquired from patients treated with primary percutaneous coronary intervention (PCI), but likely also applies to those receiving thrombolytic therapy. Failure to restore myocardial perfusion is associated with larger myocardial infarcts, increased risk of ventricular arrhythmias, cardiac failure, cardiac rupture, cardiogenic shock, major adverse cardiac events following PCI, and death. In its most extreme form, failure of microvascular perfusion is manifested in the cardiac catheterisation laboratory as the "no reflow" phenomenon; however, "slow reflow" and even more subtle degrees of impaired microvascular flow are more frequent. Longer duration from onset of symptoms and anterior myocardial infarction are risk factors for myocardial malperfusion. In addition, we have observed that diabetes mellitus is an independent predictor of diminished myocardial perfusion. ${ }^{3}$ This article provides a brief overview of the pathophysiology, diagnosis, and management of myocardial malperfusion in patients with AMI.

\section{PATHOPHYSIOLOGY}

The precise pathophysiologic mechanisms underlying myocardial malperfusion following the restoration of epicardial blood flow are unknown, and are likely to be multifactorial. Several abnormal processes have been proposed to account for impaired myocardial perfusion. ${ }^{4}$ Microvascular dysfunction is a well documented component, but the extent to which "reperfusion injury" (which implies a detrimental impact of reperfusion per se) is clinically relevant remains unclear.

First, endothelial cell integrity is pivotal for normal vascular function and ischemia-reperfusion injury is accompanied by endothelial dysfunction. Endothelial dysfunction is likely to result in reduced microvascular blood flow caused by a relative deficiency of endothelium derived vasodilators such as nitric oxide, and excess production of potent vasoconstrictors such as endothelin and oxygen free radicals. Injury to the endothelium also promotes a procoagulant and proinflammatory milieu caused by the activation of platelets, the coagulation cascade, neutrophils, and adhesion molecules which are recognised mediators of myocardial damage. In addition, cellular (endothelium, myocytes) and interstitial oedema together with cell contraction may result in occlusion of the capillaries.

Second, oxidative stress resulting from the generation of excessive oxygen free radicals from molecular oxygen entering the ischaemic myocardium and via enzymatic pathways (for example, xanthine oxidase), accompanied by a reduction in the activity of endogenous free radical scavenging pathways (for example, superoxide dismutase), likely represents an important mechanism for vascular and myocardial injury.

Third, ischaemia-reperfusion leads to increased myocardial cell calcium because of alteration in sarcolemmal calcium handling which activates calcium dependent proteases that promote damage to the myofibrils.

Finally, microembolisation of atherothrombotic material following reperfusion therapy may result in microvascular plugging. This mechanism may occur more frequently following mechanical reperfusion due to the instrumentation of the culprit coronary artery.

\section{DIAGNOSIS}

Several diagnostic tests are available to assess for the integrity of the microcirculation following successful epicardial reperfusion. In the cardiac catheterisation laboratory, a coronary Doppler flow wire can be used to detect early systolic retrograde flow, shorter systolic and diastolic flow duration, and lower coronary flow reserve in patients with TIMI 3 flow. A coronary flow reserve $<1.5$ after stent deployment during PCI is likely to be associated with $<50 \%$ ST segment elevation resolution (STR). ${ }^{5}$ An increase in coronary wedge pressure $(>20 \mathrm{~mm} \mathrm{Hg})$, measured as the distal pressure with a pressure wire, during complete epicardial occlusion with a balloon may also predict microvascular obstruction. ${ }^{6}$ However, there is limited experience with both the coronary and pressure wires for this purpose compared to angiographic indices of microvascular flow such as myocardial blush grade (MBG) and corrected TIMI frame count (CTFC). ${ }^{7} \mathrm{MBG}$ is a measure of contrast opacification of the myocardium supplied by the infarct related artery. CTFC is the number of frames required for contrast to reach a standardised distal coronary landmark.

MBG and CTFC are independent predictors of adverse outcomes in patients with TIMI 3 flow; however, in a direct comparison MBG appeared to be more powerful. ${ }^{8}$

Another well validated, non-invasive measure of myocardial perfusion following epicardial reperfusion is the electrocardiographic index of STR. ${ }^{9}$ STR is also an independent

\footnotetext{
Abbreviations: $A M I$, acute myocardial infarction; CTFC, corrected TIMI frame count; $M B G$, myocardial blush grade; $M C E$, myocardial contrast echocardiography; MRI, magnetic resonance imaging; PCl, percutaneous coronary intervention; RISK, reperfusion injury salvage kinase; STR, ST segment elevation resolution; TIMI, thrombolysis in myocardial infarction
} 
predictor of infarct size and mortality and its use in conjunction with angiographic indices of MBG or CTFC increases the predictive power. ${ }^{810}$ Myocardial contrast echocardiography (MCE) has been available for a many years for the assessment of myocardial perfusion, and the recent availability of intravenous contrast agents has made it feasible to use it for non-invasive imaging. ${ }^{2}$ The absence of perfusion by MCE correlates well with the angiographic and electrical indices described above and predicts adverse outcomes.

Cardiac magnetic resonance imaging (MRI) is the newest modality being investigated for the assessment of microvascular perfusion and appears to show great potential. The choice between these tests will depend on the availability, physician preference, and the timing of the test. Invasive, angiographic, and electrocardiographic measures would be applicable in patients undergoing primary or rescue PCI. STR analysis, MCE, and MRI would be appropriate outside the cardiac catheterisation laboratory, especially in patients treated with thrombolytics. Since AMI is a common condition requiring treatment at any time of the day, the ideal test for assessing microvascular reperfusion should be easily available, simple to use, relatively inexpensive, and easily integrated into the clinical practice. STR analysis satisfies these requirements and can be considered for routine clinical use. However, it must be emphasised that although STR is a powerful independent predictor of prognosis, the presumption that it is a surrogate measure of myocardial perfusion is based largely on clinical observations and associations.

\section{TREATMENT}

The clinical agenda has been extensive, comprising the use of multiple pharmacological and mechanical approaches. The results are impressive in that no trial has shown efficacy with respect to the predetermined primary end point. Strategies tested include a monoclonal antibody against the CD 18 subunit of the $\beta 2$ integrin adhesion receptors, endovascular cooling, hyperoxaemic perfusion therapy, and distal protection. The findings of the EMERALD trial with regards to the lack of efficacy of the Guardwire distal protection device in primary PCI are disappointing and are likely to limit the use of this approach. Nonetheless, subset analyses have suggested that agents such as adenosine, ${ }^{11}$ pexilizumab (a complement inhibitor), endovascular cooling, and hyperoxia may show some promise. Pharmacological agents that have shown promise in small clinical studies include nicorandil, a hybrid ATP sensitive potassium channel $\left(\mathrm{K}_{\mathrm{ATP}}\right)$ opener and nitric oxide donor, ${ }^{12}$ and calcium channel antagonists. ${ }^{13}$ There are conflicting data regarding the efficacy of glycoprotein IIb/ IIIa inhibitors in improving microvascular flow, ${ }^{14}{ }^{15}$ though recent data suggest that a high platelet glycoprotein IIb/IIIa receptor occupancy ( $>80 \%$ ) may improve myocardial perfusion. ${ }^{16}$ The use of stents during primary PCI is associated with a neutral effect on the microcirculation.

Prevention of microvascular injury may represent the most effective current strategy for the management of myocardial malperfusion. Several studies indicate that the incidence of this phenomenon rises with increasing delay between the onset of symptoms and restoration of epicardial flow. Thus, adherence to current guidelines with early recognition of symptoms and rapid administration of reperfusion therapy is critical. Furthermore, one can speculate that mechanical revascularisation, which accelerates myocardial reperfusion, ${ }^{17}$ may be superior to thrombolytics in patients with anterior AMI and diabetics who are greatest risk for developing myocardial malperfusion.

There are several potential explanations for the contrast between the excellent results achieved in animal models of ischaemia-reperfusion and the universally disappointing outcomes of clinical trials. These include the presence of atherosclerosis in humans, the different time scale of an evolving myocardial infarction as opposed to the release of a ligature in experimental models, the presence and extent of collaterals, other species differences, and the clinical relevance of reperfusion injury. Moreover in many animal models, agents that have the potential to modify myocardial perfusion are given before or immediately after the onset of reperfusion, a scenario that is very different to the clinical setting. Thus, the lack of benefit of the strategies tested in humans may either relate to true inefficacy or to poor trial design. It is conceivable that efforts to improve myocardial perfusion may be unsuccessful due to an inadequate therapeutic window. Patients who present early, within three hours of onset of symptoms, have an excellent prognosis with minor microvascular injury and are unlikely to benefit further from additional adjunctive treatment, while those who present later may have sustained extensive, irreversible microvascular injury.

As we reach a limit in our ability to improve epicardial patency in AMI, future research needs to focus on improving myocardial perfusion once TIMI 3 flow has been restored. Several trials are currently underway to investigate novel therapeutic targets, such as inhibition of vascular endothelial growth factor, with the aim of reducing microvascular injury. Other potential therapeutic targets include the reperfusion injury salvage kinase (RISK) pathway which may protect the myocardium from apoptotic cell death during reperfusion. ${ }^{18}$ Finally, we recommend that future clinical trials of AMI should routinely include measures of myocardial perfusion as an end point in order to evaluate the impact of new treatment strategies on reperfusion injury and the microcirculation.

\section{Authors' affiliations \\ A Prasad, B J Gersh, Division of Cardiovascular Medicine, Mayo Clinic and Mayo Foundation, Rochester, Minnesota, USA}

Correspondence to: Abhiram Prasad, MD, Mayo Clinic, 200 First Street SW, Rochester, MN 55905, USA; prasad.abhiram@mayo.edu

\section{REFERENCES}

1 Prasad A, Stone GW, Aymong E, et al. Impact of ST-segment resolution after primary angioplasty on outcomes after myocardial infarction in elderly patients: an analysis from the CADILLAC trial. Am Heart J 2004; 147:669-75.

2 Ito H, Tomooka T, Sakai N, et al. Lack of myocardial perfusion immediately after successful thrombolysis: a predictor of poor recovery of left ventricular function in anterior myocardial infarction. Circulation 1992;85:1699-705.

3 Prasad A, Stone GW, Stuckey TD, et al. Impact of diabetes mellitus on myocardial perfusion after primary angioplasty in patients with acute myocardial infraction. J Am Coll Cardial 2005;45:508-14.

4 Verma S, Fedak PW, Weisel RD, et al. Fundamentals of reperfusion injury for the clinical cardiologist. Circulation 2002;105:2332-6.

5 Feldman L, Coste P, Furber A, et al. Incomplete resolution of ST-segmen elevation is a marker of transient microcirculatory dysfunction after stenting for acute myocardial infarction. Circulation 2003;107:2684-9.

6 Sezer M, Nisanci Y, Umman B, et al. New support for clarifying the relation between ST segment resolution and microvascular function: degree of ST segment resolution correlates with the pressure derived collateral flow index. Heart 2004;90:146-50.

7 Gibson CM, Schomig A. Coronary and myocardial angiography: angiographic assessment of both epicardial and myocardial perfusion. Circulation 2004; 109:3096-105

8 Haager PK, Christott P, Heussen N, et al. Prediction of clinical outcome after mechanical revascularization in acute myocardial infarction by markers of myocardial reperfusion. J Am Coll Cardiol 2003;41:532-8.

9 Schroder R, Dissmann R, Bruggemann T, et al. Extent of early ST-segment elevation resolution: a simple but strong predictor of outcome in patients with acute myocardial infarction. J Am Coll Cardiol 1994;24:384-91.

10 Poli A, Fetiveau R, Vandoni $P$, et al. Integrated analysis of myocardial blush and ST-segment elevation recovery after successful primary angioplasty: realtime grading of microvascular reperfusion and prediction of early and late recovery of left ventricular function. Circulation 2002;106:313-18.

11 Ross A, Gibbons R, Kloner RA, et al. Acute myocardial infarction study of adenosine (AMISTAD II). J Am Coll Cardiol 2002;39(suppl A):338A.

12 Ikeda N, Yasu T, Kubo N, et al. Nicorandil versus isosorbide dinitrate as adjunctive treatment to direct balloon angioplasty in acute myocardial infarction. Heart 2004;90:181-5. 
13 Taniyama $\mathrm{Y}$, Ito $\mathrm{H}$, Iwakura $\mathrm{K}$, et al. Beneficial effect of intracoronary verapamil on microvascular and myocardial salvage in patients with acute myocardial infarction. J Am Coll Cardiol 1997;30:1193-9.

14 Costantini CO, Stone GW, Mehran R, et al. Frequency, correlates, and clinical implications of myocardial perfusion after primary angioplasty and stenting, with and without glycoprotein Ilb/llla inhibition, in acute myocardial infarction. J Am Coll Cardiol 2004;44:305-12.

15 Antoniucci D, Migliorini A, Parodi G, et al. Abciximab-supported infarct artery stent implantation for acute myocardial infarction and long-term survival: a prospective, multicenter, randomized trial comparing infarct artery stenting plus abciximab with stenting alone. Circulation 2004; 109:1704-6.
16 Gibson CM, Jennings LK, Murphy SA, et al. Association between platelet receptor occupancy after eptifibatide (Integrilin) therapy and patency, myocardial perfusion, and ST-segment I resolution among patients with STsegment-elevation myocardial infarction: an INTEGRITI (Integrilin and tenecteplase in acute myocardial infarction) substudy. Circulation 2004; 10:679-84.

17 Zeymer U, Schroder R, Machnig T, et al. Primary percutaneous transluminal coronary angioplasty accelerates early myocardial reperfusion compared to thrombolytic therapy in patients with acute myocardial infarction. Am Heart J 2003;146:686-91.

18 Hausenloy DJ, Yellon DM. New directions for protecting the heart against ischaemia-reperfusion injury: targeting the reperfusion injury salvage kinase (RISK)-pathway. Cardiovasc Res 2004;61:448-60.

\section{IMAGES IN CARDIOLOGY}

doi: $10.1136 /$ hrt. 2004.059600

Giant left ventricular thrombus after radiofrequency ablation of post-infarction ventricular tachycardia: what to do?

A

left ventricular (LV) thrombus may develop as a complication of acute myocardial infarction, ventricular aneurysm, dilated cardiomyopathy, tako-tsubo transient apical ballooning, and myocarditis. Giant LV thrombi are extremely rare and have not been previously described following radiofrequency catheter ablation (RFCA). We present a case of a giant LV thrombus developing late after an RFCA procedure for ventricular tachycardia in a patient with a post-infarction LV aneurysm. An echocardiogram performed one month after discharge showed a large, homogenously dense, and slightly oscillating mass $(28 \times 21 \mathrm{~mm})$, partially attached to the dyskinetic LV apex. With the diagnosis of giant LV thrombus, the patient began full anticoagulation treatment. Two dimensional echocardiography is an accurate non-invasive technique for the detection and follow up of LV thrombi. Factors such as the shape, size, mobility, and attachment of the thrombus to the subjacent LV myocardium influence the risk of embolic complications. One month later, on a repeat echocardiogram,

the mass had slightly reduced its size $(23 \times 14 \mathrm{~mm})$ showing ample oscillations. Oral anticoagulation treatment was intensified but two weeks later the thrombus was completely dislodged, occluding both iliac arteries. Anticoagulation treatment is not routinely recommended after LV RFCA procedures. Patients undergoing complex ablation interventions of left ventricular tachycardias associated with an aneurysm or severe left ventricular dysfunction might benefit from temporary oral anticoagulation to prevent thrombus formation. More importantly, an LV thrombus that on repeat transthoracic echocardiographic examinations becomes protruding and mobile should prompt us to consider intensive intravenous heparin, thrombolysis, or surgical thrombectomy.

\section{J Benezet-Mazuecos P Marcos-Alberca J Farre jbenezet@yahoo.es}
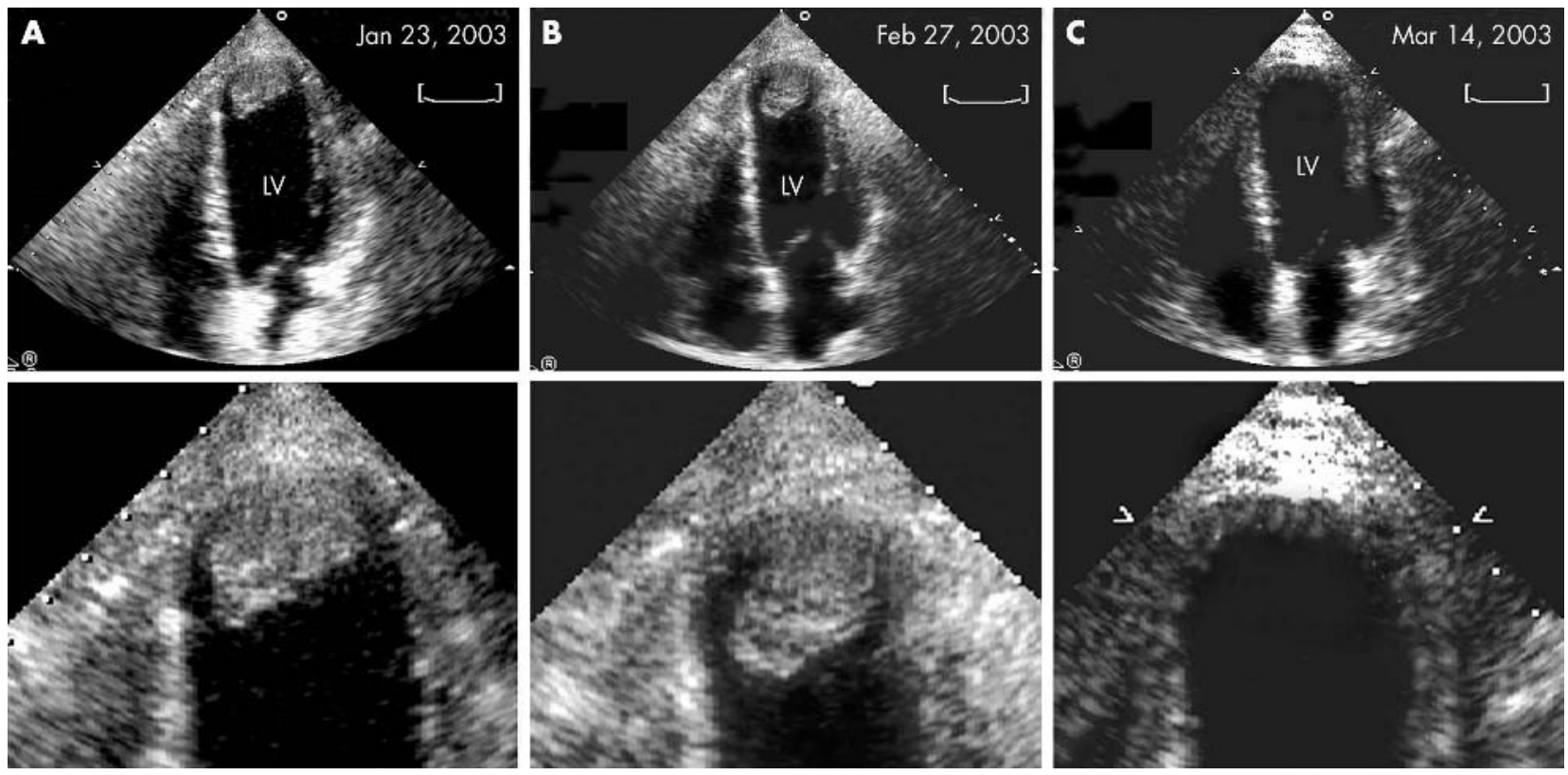

Panel A: demonstration of a giant thrombus filling the left ventricular apical aneurysm. Panel B: after one month of full oral anticoagulation treatment the thrombus was reduced in size, particularly at its attachment to the apex of the left ventricle, becoming more mobile than before. Panel C: echocardiogram obtained immediately after the embolic episode in the lower extremities showing the absence of thrombus in the left ventricle. 